\title{
BMJ Open Modifying maternal sleep position in the third trimester of pregnancy with positional therapy: a randomised pilot trial
}

\author{
Allan J Kember, ${ }^{1,2,3}$ Heather M Scott, ${ }^{4}$ Louise M O'Brien, ${ }^{5,6}$ Ali Borazjani, ${ }^{3,7}$ \\ Michael B Butler, ${ }^{1}$ Jesse H Wells, ${ }^{8}$ Andre Isaac, ${ }^{9}$ Kaishin Chu, ${ }^{10}$ Jerry Coleman, ${ }^{11,12}$ \\ Debra L Morrison ${ }^{13,14}$
}

To cite: Kember AJ, Scott HM, O'Brien LM, et al. Modifying maternal sleep position in the third trimester of pregnancy with positional therapy: a randomised pilot trial. BMJ Open 2018;8:e020256. doi:10.1136/ bmjopen-2017-020256

- Prepublication history and additional material for this paper are available online. To view these files, please visit the journal online (http://dx.doi org/10.1136/bmjopen-2017 020256).

Received 5 November 2017 Revised 24 May 2018 Accepted 22 June 2018
Check for updates

(C) Author(s) (or their employer(s)) 2018. Re-use permitted under CC BY-NC. No commercial re-use. See rights and permissions. Published by BMJ.

For numbered affiliations see end of article.

Correspondence to

Dr Allan J Kember;

allan.kember@dal.ca

\section{ABSTRACT}

Objective To evaluate whether the percentage of time spent supine during sleep in the third trimester of pregnancy could be reduced using a positional therapy device (PrenaBelt) compared with a sham device.

Design A double-blind, randomised, sham-controlled, cross-over pilot trial.

Setting Conducted between March 2016 and January 2017, at a single, tertiary-level centre in Canada.

Participants 23 participants entered the study. 20 participants completed the study. Participants were lowrisk, singleton, third-trimester pregnant women aged 18 years and older with body mass index $<35 \mathrm{~kg} / \mathrm{m}^{2}$ at the first antenatal appointment for the index pregnancy and without known fetal abnormalities, pregnancy complications or medical conditions complicating sleep. Interventions A two-night, polysomnography study in a sleep laboratory. Participants were randomised by computer-generated, one-to-one, simple randomisation to receive either a PrenaBelt or a sham-PrenaBelt on the first night and were crossed over to the alternate device on the second night. Allocation concealment was by unmarked, security-tinted, sealed envelopes. Participants, the recruiter and personnel involved in setting up, conducting, scoring and interpreting the polysomnogram were blinded to allocation.

Primary and secondary outcome measures The primary outcome was the percentage of time spent supine during sleep. Secondary outcomes included maternal sleep architecture, respiration, self-reported sleep position and feedback.

Results The median percentage of sleep time supine was reduced from $16.4 \%$ on the sham night to $3.5 \%$ on the PrenaBelt night (pseudomedian $=5.8, p=0.03$ ). We were unable to demonstrate differences in sleep architecture or respiration. Participants underestimated the time they spent sleeping supine by $7.0 \%$, and six (30\%) participants indicated they would make changes to the PrenaBelt. There were no harms in this study.

Conclusions This study demonstrates that the percentage of sleep time supine during late pregnancy can be significantly reduced via positional therapy.

Trial registration number NCT02377817; Results.

\section{Strengths and limitations of this study}

- A double-blind, randomised, sham-controlled, cross-over pilot trial.

- Employed polysomnography, the gold-standard test for investigating sleep physiology and pathophysiology.

- The first to investigate positional therapy during sleep in pregnancy.

- Performed in a controlled sleep laboratory setting over two nights; therefore, caution should be taken when extrapolating the results to the home setting and across the third trimester.

- Due to the small sample, it may have been underpowered.

\section{INTRODUCTION}

\section{Background}

Stillbirth (SB) and low birth weight (LBW) are devastating complications of pregnancy. Recently, five studies have demonstrated an association between maternal supine sleep position in late pregnancy and the risk of late term $\mathrm{SB}^{1-5}$ and LBW. ${ }^{1}$ The population attributable risk of supine sleep for SB has been reported as being between $3.7 \%$ and $37 \%,{ }^{1-5}$ suggesting that a significant proportion of late term SB could be averted if supine sleep was avoided. A number of major risk factors for SB and LBW are not modifiable in the course of the pregnancy (eg, elevated body mass index (BMI), advanced maternal age); however, maternal supine sleep is potentially modifiable with the majority of third-trimester pregnant women spending up to $25 \%$ of their sleep time supine. ${ }^{6-8}$

The contribution of supine sleep to LBW and SB is biologically plausible due to inferior vena cava compression by the gravid uterus, affecting maternal haemodynamics ${ }^{9-22}$ and fetal oxygenation. ${ }^{14} 23-25$ The supine 

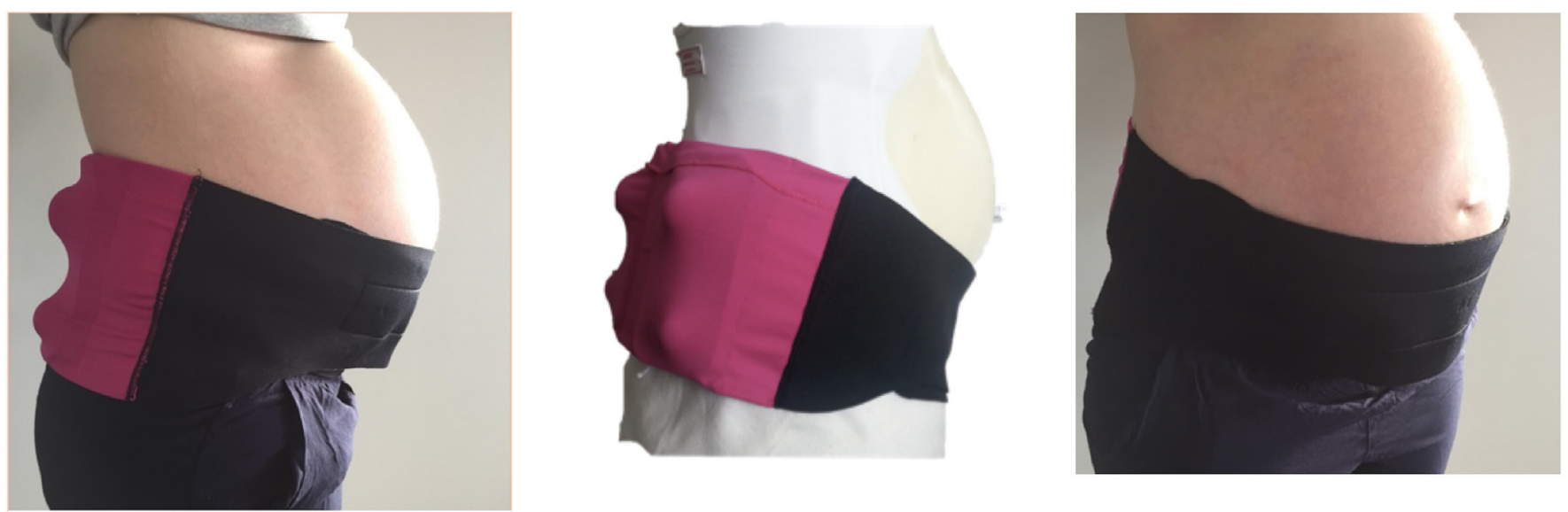

\section{Front}

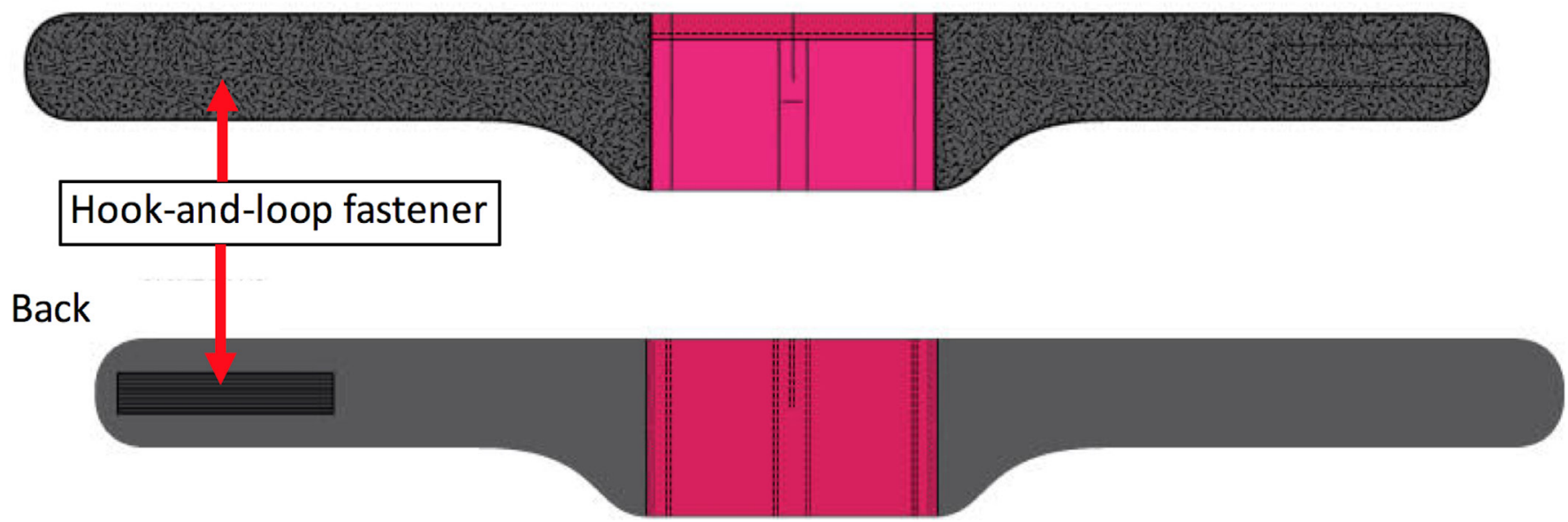

Figure 1 Photos of the PrenaBelt (left side, back and front view) and a schematic showing the PrenaBelt laid flat (front and back view).

position also exacerbates sleep-disordered breathing (SDB), ${ }^{26}{ }^{27}$ which has been linked to adverse pregnancy outcomes. ${ }^{28-30}$ In persons with mild to moderate SDB, the majority experience most of their breathing abnormalities while sleeping supine. ${ }^{31}$ Positional therapy (PT) is a simple, safe and effective treatment that helps individuals with SDB maintain a lateral position while sleeping, thus significantly reducing or eliminating their breathing abnormalities. ${ }^{3132}$ Drawing on the concept of PT for SDB, the authors (AJK, $\mathrm{AB}$ and $\mathrm{KC}$ ) designed a PT device for pregnant women called 'PrenaBelt' to minimise supine sleep (figure 1). This study is a randomised pilot trial because it trialled a new intervention on a smaller scale (pilot) to evaluate it for use in a full-scale randomised controlled trial. ${ }^{33}$

\section{Objectives}

The primary objective was to evaluate whether the percentage of time spent supine during sleep in the third trimester of pregnancy could be reduced using the PrenaBelt when compared with a sham. Secondary objectives were to evaluate the effect of the PrenaBelt on maternal sleep architecture and respiration in comparison with a sham, evaluate the accuracy of maternal self-reported sleep position and collect feedback from the participants regarding the PrenaBelt.

\section{METHODS}

\section{Randomised pilot trial design}

A single-centre, double-blind, randomised (one-to-one), sham-controlled, cross-over trial. No methodological changes were made after trial commencement.

\section{Patient and public involvement}

Patients and the public were not involved in the development of the research question or outcome measures, design of the study, recruitment process, or conduct of the study. During the consent process, participants indicated whether they wished to receive a copy of their personal study results and/or a summary of the overall study results (to be shared by email).

\section{Participants}

Participants were recruited by a trained nurse at the IWK Health Centre-a tertiary and primary care centre for women and children with approximately 5000 newborns delivered per year. Overnight polysomnograms (PSGs) 
were conducted at the Queen Elizabeth II (QEII) Health Sciences Centre Sleep Disorders Clinic. The IWK and QEII are teaching hospitals associated with Dalhousie University in Halifax, Canada. Halifax has a population of approximately 400000 . Participants expressing interest in the study (paper flyer, online advertisement) were screened.

Participants were eligible if they had a low-risk singleton pregnancy and were 18 years of age or older, more than 20 weeks gestation at screening, and residing in the Halifax Regional Municipality. Exclusion criteria included BMI $\geq 35 \mathrm{~kg} / \mathrm{m}^{2}$ at the first antenatal appointment for the current pregnancy, pregnancy complicated by obstetric conditions (eg, hypertension, diabetes, intrauterine growth restriction), sleep complicated by any medical conditions (eg, known obstructive sleep apnoea (OSA), insomnia, musculoskeletal deformity affecting sleep position), multiple pregnancy, known fetal abnormality, and non-English speaking and reading.

There was no independent data monitoring committee.

\section{Interventions}

There was no run-in measurement of baseline sleep habits. Each participant underwent two overnight PSG studies between 28 and 37 weeks gestation. The study nights were not required to be consecutive dates, and we did not specify a defined washout period. The only difference between the two nights was the intervention (PrenaBelt or sham) and that participants completed a demographic questionnaire on the first PSG night only. The PrenaBelt is worn at the level of the waist and has four firm plastic balls embedded into foam inserts within the pockets posteriorly (figure 1). The mechanism of action of the PrenaBelt is theoretical and based on the tennis ball technique of PT, ${ }^{34-36}$ when supine, the balls apply pressure points across the user's lower back, prompting her to reposition herself in a lateral position to maintain comfort. The sham was identical in appearance, materials and construction to the PrenaBelt, but had soft foam balls instead of firm plastic balls and did not have foam inserts; as such, the sham did not provide pressure points. Participants crossed over to the alternative intervention on the second PSG night.

PSG set-up, including Pro-Tech zRIP Durabelts (Philips Respironics, Murrysville, Pennsylvania, USA) for respiration, PT1 pressure transducers (BRAEBON Medical, Kanata, Canada) for airflow and snoring, electrodes (Natus Medical, Pleasanton, California, USA) for ECG/ electroencephalography (EEG)/electrooculography/ electromyography and finger-tip pulse oximetry, was by trained research assistants (RAs), in accordance with the American Academy of Sleep Medicine 2014 guidelines, ${ }^{37}$ and in a private clinical room with continuous monitoring by an RA in a separate room through audio/visual and Embla Sandman Elite sleep diagnostic software.

Immediately before the participant settled to sleep, the unblinded research personnel (co-PI (AJK) or study coordinator (JHW)) entered the room to assist her in donning the assigned intervention (PrenaBelt/sham) without disclosing the identity of the intervention to the participant or the RA in order to maintain blinding. On waking for the day, the participants immediately removed the intervention and placed it in an opaque closed box for collection by the unblinded personnel in order to keep it concealed from the RA. The RA subsequently entered the room and unhooked the PSG equipment. The participant completed a feedback questionnaire and placed it in a sealed envelope for the unblinded personnel. Participants were permitted bathroom breaks and to remove the intervention and/or PSG equipment at any point if they became uncomfortable.

Scoring of PSG's was performed by blinded Registered PSG Technicians, and interpretation was by a blinded sleep medicine physician (DLM).

\section{Outcomes}

The prespecified primary outcomes per protocol were the per cent of time spent in the supine and right-lateral positions (we originally intended to minimise both) and the feedback questionnaire; however, for reporting clarity and participant comfort, the PrenaBelt was configured to provide pressure points only when the user was supine, the primary outcome was further specified to be the percentage of time spent supine during sleep only, and the other prespecified primary outcomes were instead reported as secondary outcomes. The primary outcome was documented continuously by an RA via video feed.

Secondary outcomes were total sleep time, sleep time in each position (left lateral, right lateral, supine, prone), percentage of sleep left and right, sleep latency, sleep efficiency, sleep indices (total arousal, spontaneous arousal, periodic limb movement, respiratory arousal, apnoeahypopnea, respiratory effort-related arousal), percentage of sleep stage (1, 2, 3 and rapid eye movement (REM)), peripheral oxygen saturation $\left(\mathrm{SpO}_{2}\right)$ during sleep, snoring and feedback questionnaire. The questionnaire elicited participants' perceptions of body position at sleep onset and waking, number of changes in position, and estimates of percentage/time spent in each position during sleep. Participants also ranked their satisfaction, comfort level and intention to continue using the PrenaBelt (if it were available) on a 10-point Likert scale (with 10 out of 10 reflecting the highest satisfaction, comfort and intention to use, and 1 out of 10 the lowest).

Per our research protocol and trial registry, supine and non-supine Apnoea-Hypopnea Index (AHI), min/ mean/max $\mathrm{SpO}_{2}$ while awake and $\mathrm{min} / \mathrm{mean} / \mathrm{max}$ maternal heart rate during wake, REM and non-REM (NREM) states, were also specified as secondary outcomes; however, we were unable to report the supine and non-supine AHI and heart rate data due a software configuration issue nor the awake $\mathrm{SpO}_{2}$ values due to data artefact.

\section{Sample size}

This is the first trial investigating PT in pregnant women to reduce supine sleep time. For a one-sided paired 


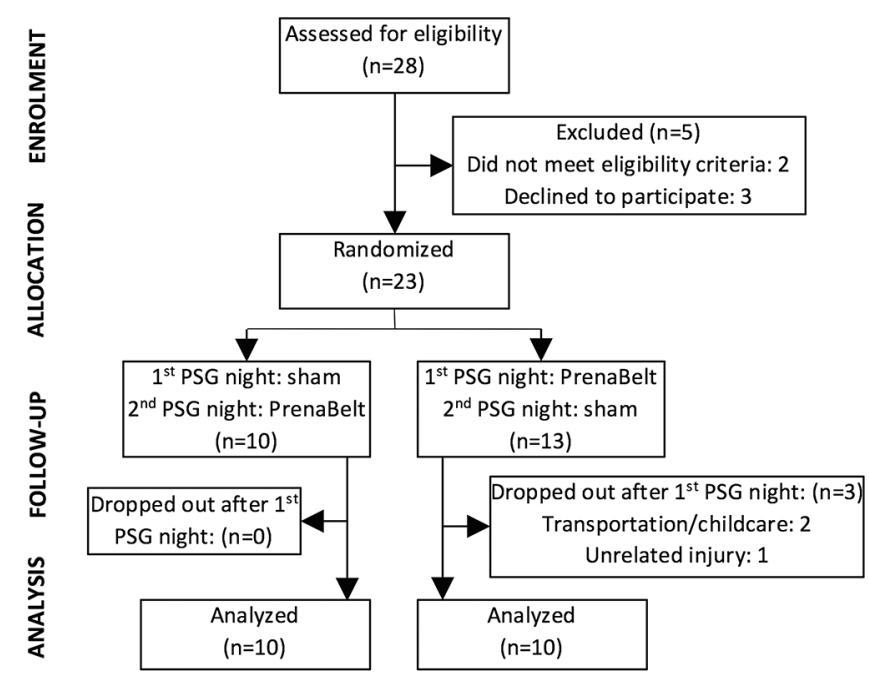

Figure 2 Enrolment, allocation and analysis of trial participants. PSG, polysomnogram.

t-test with power $(\beta)$ of 0.80 , significance level $(\alpha)$ of 0.05 and detectable effect $(d)$ of -0.5 (difference in mean percentage of time spent sleeping supine by half an SD), which is a medium effect size per the literature regarding Cohen, ${ }^{38}$ a sample size of $25(n=25)$ participants was required. All statistical analyses were conducted using the R Statistical Software package (V.3.2.4 (2016-0310)). ${ }^{39}$ There was no planned interim analysis or stopping guidelines.

\section{Randomisation}

Participants were allocated by computer-generated (R), one-to-one, simple randomisation into one of two possible cross-over schemes: PrenaBelt on the first PSG night followed by sham on the second PSG night and vice versa (figure 2). Allocation sequence was concealed via unmarked, security-tinted, sealed envelopes by an independent statistician (MBB). An envelope was drawn in sequence, opened, and the participant's name and birth date were recorded on the enclosed allocation sheet by the unblinded personnel (AJK, JHW) on the participant's first PSG night.

\section{Blinding}

Participants, the recruiter and personnel involved in setting up, conducting, scoring and interpreting the PSG were blinded to allocation. The only research personnel unblinded to the allocation were the co-PI (AJK) and the study coordinator (JHW), which was required in order to provide the appropriate intervention to the participant before she went to sleep. It was not feasible to blind the researchers analysing the study data (AJK, JHW, MBB) to the allocation.

\section{Statistical methods}

Primary and secondary outcomes were compared between interventions by paired t-tests (normal) and Wilcoxon signed-rank tests (non-normal). Cohen's Kappa ( $\kappa)$ was used to assess agreement between categorical variables.
Spearman's $r$ and Bland-Altman plots were used for continuous variables.

For continuous variables, normality was assessed using Q-Q plots and Anderson-Darling test. All testing was performed at a 0.05 significance level. Treatment effects (differences in means and OR) and associated CIs were presented at a $95 \%$ CI with $p$ values.

\section{RESULTS}

Between March 2016 and January 2017, 28 participants were assessed for eligibility (figure 2). Following exclusion of three (11\%) who declined participation and two $(7 \%)$ who failed to meet eligibility criteria, 23 were randomised. After randomisation, there were three (13\%) drop-outs-all from the PrenaBelt-then-sham group and after completing the first PSG night. Two were due to transportation/childcare reasons, and one was due to an unrelated injury preventing her from sleeping in a horizontal position. Thus, 20 participants-10 from each group-were included in the primary analysis, which was by original assigned groups and on a complete-case basis (drop-outs excluded). The originally planned sample size of 25 participants was not reached due to unforeseen budget restrictions preventing recruitment beyond 20 participants. The length of time between studies for the same participant (washout period) was a median of 1 day (IQR 1-3.25 days; maximum 13 days).

\section{Sample characteristics}

Baseline demographic, obstetric and sleep habit characteristics of the 20 participants who completed the study are shown in table 1 per randomised group $(\mathrm{n}=10$ each). The two randomised groups were well balanced with respect to the sample characteristics-there were no statistically significant differences in baseline characteristics between groups $(\alpha=0.05)$. The mean (SD) age was $30.9(5.0)$ years. The majority $(80 \%)$ were Caucasian. The mean (SD) prepregnancy BMI was $26.6 \mathrm{~kg} / \mathrm{m}^{2}$ (3.3) and rose to a mean of $30.4 \mathrm{~kg} / \mathrm{m}^{2}(3.6)$ at the time of the first PSG, which took place at a median (IQR) gestational age of 30.0 weeks (28-33.1). Half $(50 \%)$ of the participants were nulliparous.

The median (IQR) self-reported overnight sleep duration at the time of the first PSG was 8 (7-8) hours. In the week previous to the first PSG (when pregnant), left was the most common position at sleep onset $(85 \%)$ and waking $(60 \%)$, although a large proportion of women also reported the supine position at sleep onset and waking (25\% and $40 \%$, respectively). When not pregnant, prone was the most common position at sleep onset $(65 \%)$ and supine was the most common at waking (55\%). Most participants used a pillow under their head $(90 \%)$ and between their knees $(70 \%)$.

In comparing baseline demographic, obstetric and sleep habit characteristics between the participants who completed the study $(n=20)$ and those who did not $(n=3)$, the groups were similar; however, participants who did not 
Table 1 Baseline demographic, obstetric and sleep habit characteristics

PrenaBelt-then-sham $(\mathbf{n}=10)$

\section{Sham-then-PrenaBelt $(n=10)$}

\begin{tabular}{lrr}
\hline Age (years) & $31.1(6.0)$ & $30.7(4.1)$ \\
Caucasian ethnicity & $6(60 \%)$ & $10(100 \%)$ \\
Prepregnancy BMI $\left(\mathrm{kg} / \mathrm{m}^{2}\right)$ & $27.0(3.3)$ & $26.2(3.5)$ \\
Current BMI $\left(\mathrm{kg} / \mathrm{m}^{2}\right)$ & $30.9(3.7)$ & $29.9(3.6)$ \\
Gestational age (weeks) & $30.9(3.0)$ & $30.8(2.8)$
\end{tabular}

Gravida

\begin{tabular}{|c|c|c|}
\hline 1 & $4(40 \%)$ & $6(60 \%)$ \\
\hline$\geq 2$ & $6(60 \%)$ & $4(40 \%)$ \\
\hline Sleep duration (hours) & $8.0(7.5-8.0)$ & $7.8(7.0-8.0)$ \\
\hline \multicolumn{3}{|l|}{ In the last week } \\
\hline \multicolumn{3}{|l|}{ Sleep-onset positions } \\
\hline Left & $7(70 \%)$ & $10(100 \%)$ \\
\hline Supine & $3(30 \%)$ & $2(20 \%)$ \\
\hline Right & $8(80 \%)$ & $3(30 \%)$ \\
\hline Prone & $1(10 \%)$ & $0(0 \%)$ \\
\hline \multicolumn{3}{|l|}{ Waking positions: } \\
\hline Left & $8(80 \%)$ & $4(40 \%)$ \\
\hline Supine & $4(40 \%)$ & $4(40 \%)$ \\
\hline Right & $5(50 \%)$ & $6(60 \%)$ \\
\hline Prone & $0(0 \%)$ & $0(0 \%)$ \\
\hline
\end{tabular}

When not pregnant

Sleep-onset positions:

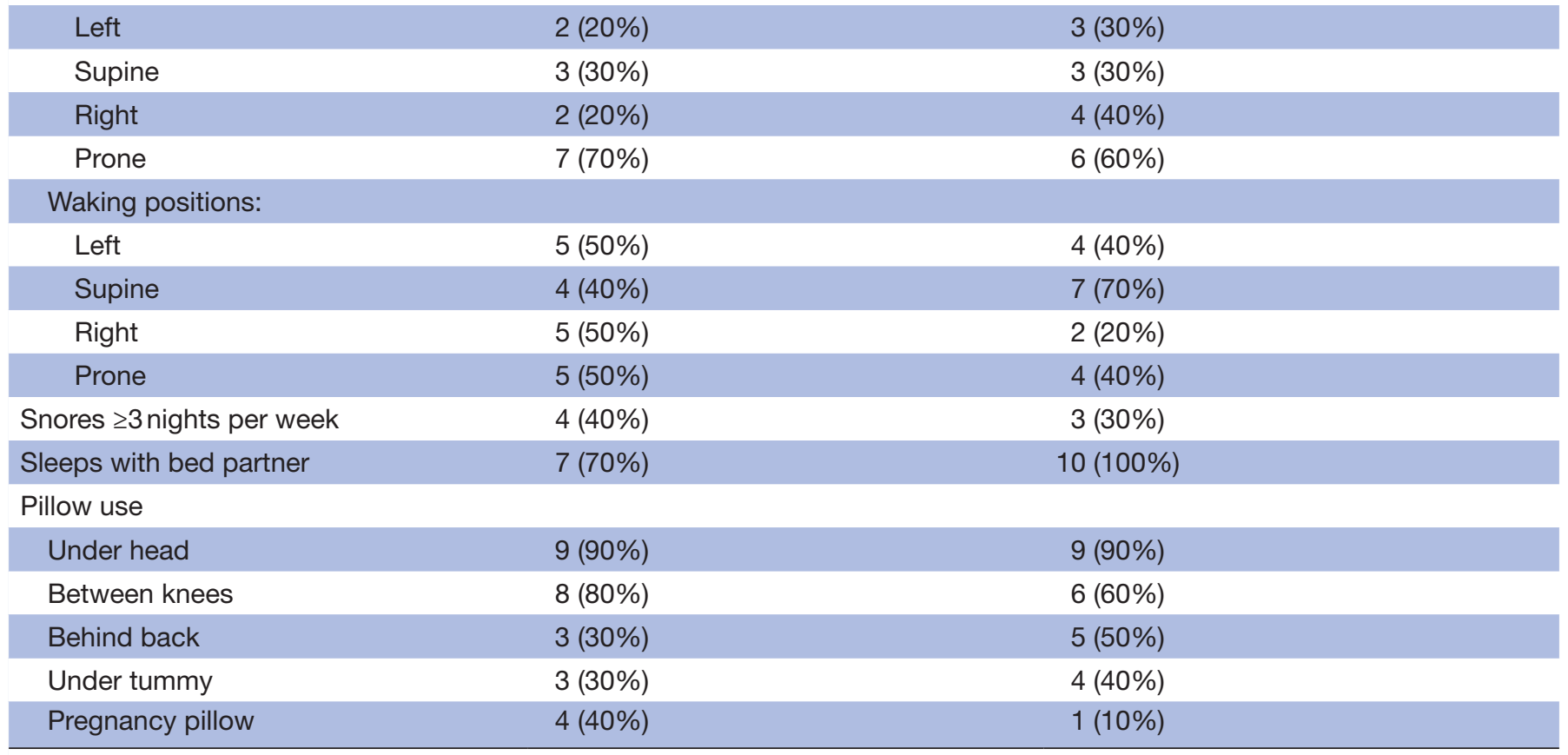

Normally distributed continuous variables are reported as mean (SD). Non-normally distributed continuous variables are presented as median (IQR). Count data are presented as frequency (\%).

Percentages for responses to some questions may add to greater than $100 \%$ because some participants checked more than one box in response to a question, for example, for sleep-onset position in the last week, some responded 'left' and 'right'.

BMI, body mass index.

complete the study were younger (mean 26.7 years), had a higher current BMI (mean $32.5 \mathrm{~kg} / \mathrm{m}^{2}$ ) and had less self-reported overnight sleep duration (mean 6.8 hours).

\section{Primary outcome}

The primary outcome was not normally distributed. The median (IQR) percentage of sleep time supine on the 
Table 2 Primary outcome: percentage (\%) of sleep time supine

\begin{tabular}{llll}
\hline & $\begin{array}{l}\text { PrenaBelt } \\
\text { night }(\mathbf{n}=20)\end{array}$ & $\begin{array}{l}\text { Sham night } \\
(\mathbf{n}=20)\end{array}$ & P values \\
\hline Median (IQR) & $3.5(0-16.6)$ & $16.4(3.5-25.3)$ & 0.03 \\
\hline
\end{tabular}

PrenaBelt night $(\mathrm{n}=20)$ and sham night $(\mathrm{n}=20)$ was $3.5 \%$ $(0-16.6)$ and $16.4 \%$ (3.5-25.3), respectively (table 2, figure 3). On a one-sided paired Wilcoxon signed-rank test, the median of the pairwise differences (pseudomedian) in percentage of sleep time supine between the sham night versus the PrenaBelt night was significantly greater than 0 (pseudomedian $=5.8 ; 95 \%$ CI 0.70 to Inf; $\mathrm{p}=0.03)$.

\section{Secondary outcomes}

The estimates, 95\% CIs and $p$ values for the intervention-associated differences, PrenaBelt versus sham, in the sleep and respiratory variables and user feedback are shown in table 3 . The median (IQR) number of minutes spent sleeping supine and right lateral was 38.9 $(0-72.7)$ and $90.8(0-166.1)$, respectively. The mean (SD) number of minutes spent sleeping left lateral was 180.9 (97.7). The mean (SD) percentage of time spent sleeping in the left-lateral and right-lateral positions was 54.6\% (26.7) and 30.3\% (27.8), respectively. No time was spent sleeping prone.

Regarding sleep architecture for the complete sample ( $\mathrm{n}=40$ nights), the median (IQR) sleep time was $352 \mathrm{~min}$ (318-374), sleep efficiency 82\% (76-89) and sleep latency $12.8 \mathrm{~min}(6.7-23.9)$. The median (IQR) total arousal index was 11.3 (8.4-18.0), spontaneous arousal index 9.8 (7.0-12.0), periodic leg movement arousal index $0(0-0.8)$ and respiratory arousal index $0.4(0-1.0)$. The mean (SD) percentage of stage 1 sleep was $9.4 \%$ (5.6), stage 2 sleep $62.7 \%$ (7.9), stage 3 sleep $11.2 \%$ (8.3) and REM sleep $16.7 \%$ (5.6). For respiratory variables, the median (IQR) AHI was $0.5(0-1.5)$, respiratory effort-related arousal index $0(0-0.2)$, Respiratory Disturbance Index

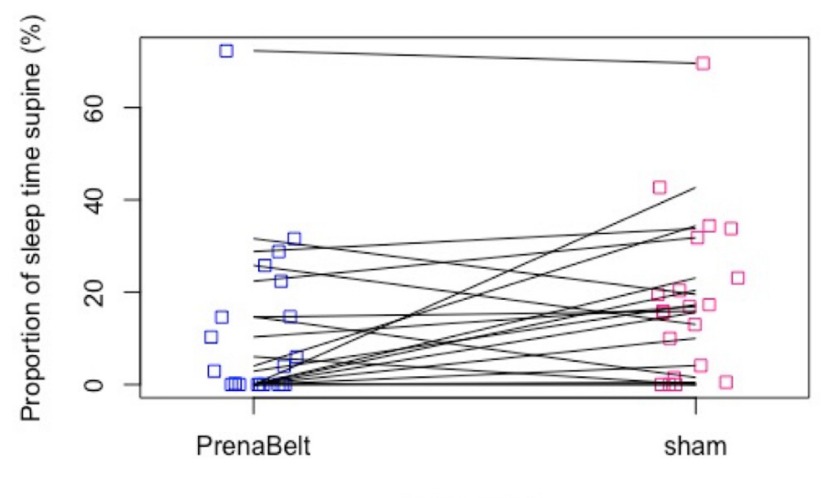

Intervention

Figure 3 Scatter plot of proportion of sleep time supine (\%) versus intervention (PrenaBelt, sham) for each participant. Each line represents one participant.
(RDI) 0.6 (0-1.6), RDI supine 0 (0-0.9), RDI non-supine 0.5 (0-1.6), $\mathrm{SpO}_{2}$ maximum and minimum during REM sleep were $98 \%$ (98-99) and 92\% (90-94), respectively, and $\mathrm{SpO}_{2}$ maximum and minimum during NREM sleep were 99\% (98-100) and 92\% (91-94), respectively. The mean (SD) $\mathrm{SpO}_{2}$ during REM and NREM sleep was 96.0 (1.2) and 96.2 (1.0), respectively. Presence of snoring was detected on 26 of $40(65 \%)$ nights. Six participants $(30 \%)$ indicated that they would make changes to the PrenaBelt—of these, five were with regard to comfort and one was with regard to sizing. There were no statistically significant differences in sleep architecture, respiration or user feedback between the PrenaBelt and sham nights on paired testing ( $\mathrm{n}=20$ pairs) (table 3 ).

The self-reported sleep data are presented in table 4 with the PSG-recorded correlates and the statistical analysis of agreement. Participants recalled their sleep-onset position accurately for 34 of 40 nights $(85 \%, \kappa 0.62$, moderate agreement). Participants recalled their waking position accurately for 28 of 40 nights $(70 \%, \kappa 0.42$, weak agreement). There was a weak relationship between sleep-onset and waking position $(\kappa 0.24)$. There was no agreement between the self-reported and PSG-reported number of position changes ( $\kappa 0.17$ ). Percentage of time in each position, left, supine and right, as estimated per self-report and measured per PSG had Spearman's $\mathrm{r}$ of 0.76 (good correlation, $\mathrm{p}<0.01$ ), 0.27 (poor correlation, $\mathrm{p}=0.11$ ), and 0.93 (excellent correlation, $\mathrm{p}<0.01$ ), respectively. Bland-Altman plots (figures 4-6) demonstrated that, on average, participants' self-reports tended to overestimate the percentage of leftside and right-side sleep by $5.5 \%$ (95\% CI -30.2 to 41.2 ) and $0.5 \%$ (95\% CI -21.9 to 22.9 ), respectively, and underestimate the percentage of supine sleep by $7.0 \%$ (95\% CI -20.1 to 34.1) when compared with the PSG-determined position.

\section{Harms}

No participants (including the drop-outs) requested to remove the intervention or PSG equipment. There were no known harms related to the interventions or procedures in this study.

\section{DISCUSSION \\ Principal findings}

Use of the PrenaBelt resulted in a reduction in the percentage of sleep time supine in comparison with the sham, and we were unable to demonstrate an effect on maternal sleep architecture or respiration.

\section{Strengths and weaknesses in relation to other studies}

Our study only included two nights for each participant and was a randomised pilot trial; therefore, caution should be taken when extrapolating our results across the third trimester with regard to efficacy and compliance-PT in non-pregnant individuals with positional OSA has sustained efficacy in the long term but decreasing compliance from $93 \%$ at 1 month, ${ }^{40} 74 \%$ at 3 months ${ }^{41}$ and $60 \%$ at 6 months. ${ }^{42}$ 
Table 3 Secondary outcomes: time supine, sleep architecture, respiration, user feedback

\begin{tabular}{|c|c|c|c|c|}
\hline & $\begin{array}{l}\text { PrenaBelt } \\
(\mathrm{n}=20)\end{array}$ & $\begin{array}{l}\text { Sham } \\
(n=20)\end{array}$ & $\begin{array}{l}\text { PrenaBelt minus sham } \\
\text { Difference }(95 \% \mathrm{Cl})\end{array}$ & $P$ values \\
\hline Total sleep time (minutes) & $353(318-376)$ & $350(318-374)$ & $-3.6^{\star}(-33.8$ to 21.8$)$ & 0.81 \\
\hline Supine sleep (minutes) & $12.3(0-53.5)$ & $56.8(12.4-79.1)$ & $-26.4^{\star}(-49.9$ to -2.6$)$ & 0.03 \\
\hline Left-lateral sleep (minutes) & $185.6(102.5)$ & $176.1(95.2)$ & $9.5+(-24.3$ to 43.3$)$ & 0.56 \\
\hline Right-lateral sleep (minutes) & $108.9(110.1)$ & $101.9(100.9)$ & $6.9 \dagger(-22.9$ to 36.7$)$ & 0.63 \\
\hline Percentage sleep left (\%) & $57.0(26.9)$ & $52.2(27.0)$ & $4.8 \dagger(-5.1$ to 14.8$)$ & 0.32 \\
\hline Percentage sleep right (\%) & $31.3(28.8)$ & $29.3(27.5)$ & $2.0 \dagger(-6.0$ to 10.1$)$ & 0.60 \\
\hline Sleep latency (minutes) & $11.7(6.7-21.9)$ & $14.5(7.2-26.2)$ & $2.0^{\star}(-7.2$ to 9.4$)$ & 0.65 \\
\hline Sleep efficiency (\%) & $81(76-89)$ & $83(79-88)$ & $-1.4^{*}(-5.9$ to 3.0$)$ & 0.46 \\
\hline Total arousal index & $11.2(8.1-18.0)$ & $12.0(8.5-15.6)$ & $0.60^{\star}(-1.5$ to 3.5$)$ & 0.59 \\
\hline Spontaneous arousal index & $10.5(5.8)$ & $10.3(5.5)$ & $0.20 \dagger(-2.2$ to 2.6$)$ & 0.86 \\
\hline PLM arousal index & $0(0-1.0)$ & $0.1(0-0.7)$ & $0.3^{*}(-1.5$ to 16.0$)$ & 0.68 \\
\hline Respiratory arousal index & $0.4(0-1.1)$ & $0.4(0-1.0)$ & $0.1^{*}(-0.5$ to 2.1$)$ & 0.78 \\
\hline Percent stage 1 sleep (\%) & $9.1(5.2)$ & $9.6(6.0)$ & $-0.50 \dagger(-2.3$ to 1.3$)$ & 0.56 \\
\hline Percent stage 2 sleep (\%) & $64.5(7.4)$ & $61.0(8.1)$ & $3.5+(-0.3$ to 7.3$)$ & 0.07 \\
\hline Percent stage 3 sleep (\%) & $10.9(8.0)$ & $11.5(8.8)$ & $-0.63 \dagger(-2.6$ to 1.4$)$ & 0.52 \\
\hline Percent REM sleep (\%) & $15.5(6.4)$ & $18(4.6)$ & $-2.5 \dagger(-5.1$ to 0.05$)$ & 0.05 \\
\hline Apnoea-Hypopnea Index & $0.5(0-1.5)$ & $0.5(0-1.5)$ & $-0.14^{*}(-0.8$ to 0.4$)$ & 0.51 \\
\hline RERA index & $0(0-0.1)$ & $0(0-0.3)$ & $0.15^{\star}(-0.4$ to 4.6$)$ & 0.78 \\
\hline Respiratory Disturbance Index & $0.65(0-1.5)$ & $0.55(0.15-1.75)$ & $0.2^{*}(-0.6$ to 1.2$)$ & 0.67 \\
\hline RDI while supine & $0(0-0)$ & $0(0-1.2)$ & $-1.3^{*}(-11.2$ to 2.4$)$ & 0.48 \\
\hline RDI while non-supine & $0.2(0-1.5)$ & $0.5(0-1.75)$ & $-0.05^{\star}(-0.85$ to 1.0$)$ & 0.92 \\
\hline \multicolumn{5}{|l|}{$\mathrm{SpO}_{2}$ during REM (\%) } \\
\hline Maximum & 98 (98-99) & 98.5 (98-99) & $0.0^{*}(-1.0$ to 0$)$ & 0.28 \\
\hline Mean & $95.8(1.2)$ & $96.2(1.2)$ & $-0.46 \dagger(-1.1$ to 0.15$)$ & 0.13 \\
\hline Minimum & $92.5(90.8-94)$ & $92(89-94)$ & $0.5^{\star}(-1.0$ to 2.5$)$ & 0.40 \\
\hline \multicolumn{5}{|l|}{$\mathrm{SpO}_{2}$ during NREM (\%) } \\
\hline Maximum & $99.0(98-100)$ & $99(98-100)$ & $0.5^{\star}(-1.0$ to 1.5$)$ & 0.46 \\
\hline Mean & $96.1(0.88)$ & $96.4(1.0)$ & $-0.29+(-0.71$ to 0.14$)$ & 0.17 \\
\hline Minimum & $92(91-94)$ & $92.5(89-94)$ & $0.5^{*}(-0.5$ to 2.5$)$ & 0.26 \\
\hline Presence of snoring & $15(75 \%)$ & $11(55 \%)$ & $2.4 \ddagger(0.54$ to 11.9$)$ & 0.32 \\
\hline Satisfaction (out of 10) & $7.5(6.0-8.3)$ & $7.5(5.5-9.0)$ & $-0.28 \dagger(-1.0$ to 0.5$)$ & 0.46 \\
\hline Comfort (out of 10) & $9.0(6.8-9.3)$ & $9.0(7.8-10)$ & $-1^{*}(-2.8$ to 0$)$ & 0.06 \\
\hline Intention to use (out of 10) & $8.0(5.8-8.0)$ & $7.0(6.0-9.0)$ & $-0.1 \dagger(-1.0$ to 0.8$)$ & 0.81 \\
\hline
\end{tabular}

P-values less than 0.05 are bold text.

Count data (presence of snoring) are presented as frequency (\%).

Units for 'index' variables are events per hour.

*Non-normally distributed continuous variables and discrete data (satisfaction, comfort and intention to use) are presented as median (IQR), and two-sided paired Wilcoxon signed-rank test is used to test for difference ('Difference $(95 \% \mathrm{Cl})$ ' is the median of the pairwise differences (pseudomedian) and $95 \% \mathrm{Cl}$ for the pseudomedian).

†Normally distributed continuous variables are reported as mean (SD), and paired t-test is used to test for difference.

fFisher's exact test ('difference' is OR).

NREM, non-rapid eye movement; PLM, periodic leg movement; RDI, respiratory disturbance index; REM, rapid eye movement; RERA, respiratory effort-related arousal; $\mathrm{SpO}_{2}$, peripheral oxygen saturation.

In recent studies of PT in non-pregnant individuals with positional OSA, PT devices incorporating active supine sensing technology and vibration mechanisms have been shown to be more effective in reducing supine sleep than inactive pressure-point PT devices like the
PrenaBelt. ${ }^{41}{ }^{43}$ In comparison to a study of pressure-point PT in non-pregnant individuals with positional OSA by Heinzer et $a l,{ }^{41}$ the PrenaBelt resulted in a lesser reduction in mean percentage of sleep time supine; however, in contrast, we did not have a non-intervention night as 
Table 4 Secondary outcomes: self-reported versus PSG-recorded sleep behaviours

\begin{tabular}{|c|c|c|c|}
\hline & Self-report & PSG & Agreement \\
\hline \multicolumn{4}{|l|}{ Sleep-onset position $(n=40)^{*}$} \\
\hline Left & $31(78 \%)$ & $29(73 \%)$ & \multirow[t]{2}{*}{ Cohen's к† 0.62} \\
\hline Right & $7(17 \%)$ & $8(20 \%)$ & \\
\hline \multicolumn{4}{|l|}{ Waking position $(n=40)^{*}$} \\
\hline Supine & $0(0 \%)$ & $5(13 \%)$ & \multirow{2}{*}{ Cohen's к† 0.42} \\
\hline Right & $12(30 \%)$ & $13(32 \%)$ & \\
\hline No of position changes $(n=30) \ddagger$ & $3(2-4)$ & $6(3.3-10)$ & Cohen's $\kappa \S 0.17$ \\
\hline \multicolumn{4}{|c|}{$\%$ of total sleep time in position $(n=35) \eta$ : } \\
\hline Left & $59.9(28.1)$ & $54.4(26.4)$ & Spearman's $\rho 0.76$ \\
\hline
\end{tabular}

${ }^{*}$ Count data are presented as: frequency (\%).

†Linear/equal weighted Cohen's $\kappa$.

‡Ordinal data presented as: median (IQR).

§Unweighted Cohen's $\kappa$.

ๆContinuous data presented as: mean (SD).

PSG, polysomnogram.

Heinzer et al did. The sham, by intrinsic characteristics, may have caused a reduction in supine sleep in comparison to a non-intervention night. O'Brien and Warland ${ }^{6}$ reported a non-interventional, one-night, in-home study of 51 healthy women in their second and third trimester using ambulatory PSG; in a subgroup of participants in their third trimester $(n=33)$, the median percentage of overall sleep time spent supine was $26.5 \%$. McIntyre $e t$ $a l,{ }^{7}$ in a similar non-interventional, one-night, in-home, ambulatory PSG study in 30 healthy women in the third trimester, reported the median percentage of overall sleep time spent supine to be $19 \%$. Participants were not asked to adopt or try to maintain any sleep position in either study. Both of these studies reported a greater

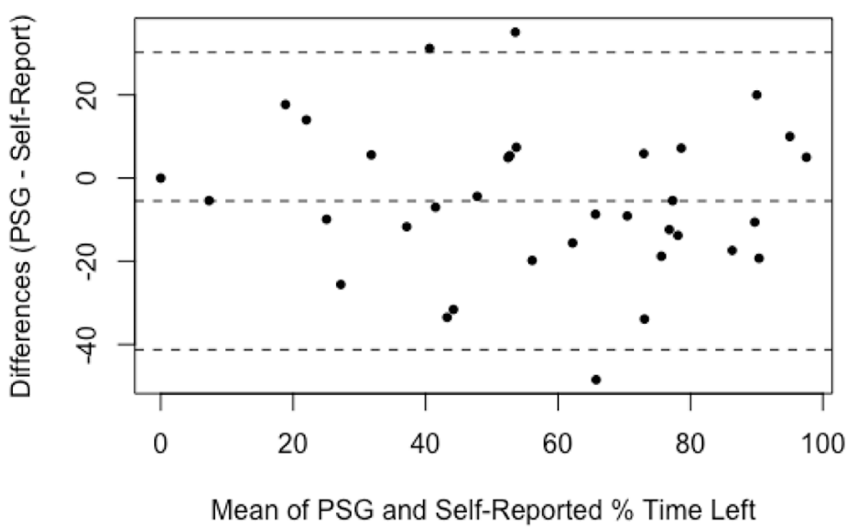

Figure 4 Bland-Altman plot for percentage of time spent sleeping on the left side; difference between self-reported estimate and PSG-determined values. Broken lines indicate mean difference and upper and lower limits of agreement (95\% Cl). PSG, polysomnogram. median percentage of overall sleep time spent supine than that in the current study $(16.4 \%)$, which indicates that the sham device may have affected position. This, along with differences inherent to pregnancy (eg, body habitus, increased sleep disruption) may account for the lesser reduction in mean percentage of sleep time supine achieved by the PrenaBelt in comparison Heinzer $e t a l .{ }^{41}$

Zaremba et al undertook a randomised, cross-over, PSG study in 30 postpartum women sleeping in non-elevated and $45^{\circ}$ elevated body position and reported moderate to severe OSA in $20 \%$ of the participants. ${ }^{44}$ The minimum $\mathrm{SpO}_{2}$ was higher ( $\mathrm{p} 0.03$ ) and AHI was reduced ( $\mathrm{p} 0.03$ )

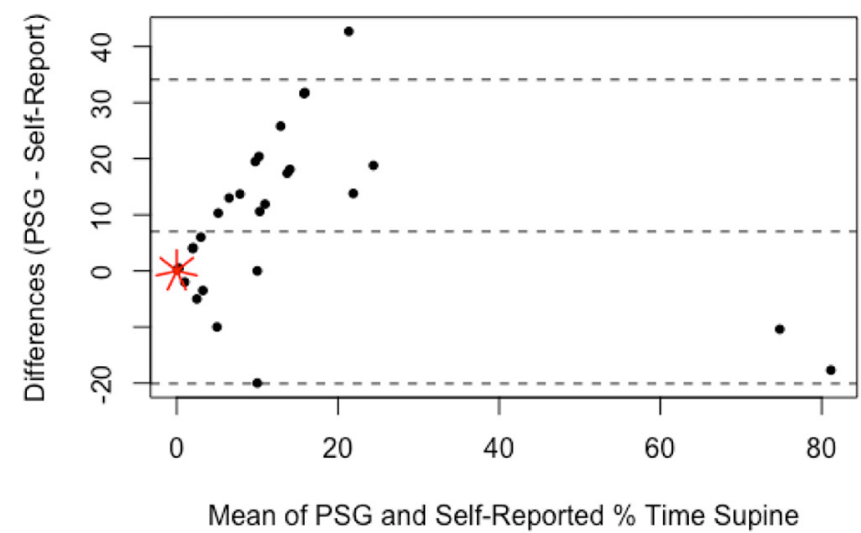

Figure 5 Bland-Altman plot for percentage of time spent sleeping supine; difference between self-reported estimate and PSG-determined values. Broken lines indicate mean difference and upper and lower limits of agreement $(95 \% \mathrm{Cl})$. Each short red line indicates an additional data point at that location (sunflower plot). PSG, polysomnogram. 


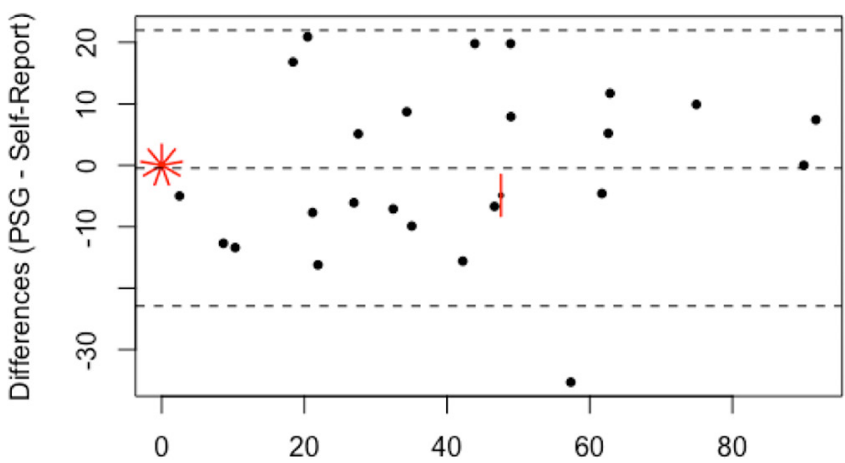

Mean of PSG and Self-Reported \% Time Right

Figure 6 Bland-Altman plot for percentage of time spent sleeping on the right side; difference between self-reported estimate and PSG-determined values. Broken lines indicate mean difference and upper and lower limits of agreement $(95 \% \mathrm{Cl})$. Each short red line indicates an additional data point at that location (sunflower plot). PSG, polysomnogram.

in the $45^{\circ}$ elevated body position in comparison to non-elevated without affecting sleep quantity or quality. In our analysis, we were unable to demonstrate any differences in sleep architecture or duration despite a significant difference in body position (less supine time).

Seventy-three per cent of participants in the study by McIntyre $e t a l^{7}$ recalled their sleep-onset position accurately ( $\kappa 0.52)$, which is a lower accuracy than our results $(85 \%$ accurate, $\kappa$ 0.62) possibly because each of our participants underwent two PSG nights and, after being asked to recall various details about their sleep after their first PSG night, may have anticipated these questions again after their second PSG night. Forty per cent of McIntyre et als participants recalled their waking position accurately $(\kappa 0.24)$, which is a lower accuracy than our results $(70 \%$ accurate, $\kappa$ 0.42). Being an ambulatory PSG, McIntyre et al's study did not include EEG - they estimated sleep and wake times by predefined video criteria (ie, lack of movement) and not by brain activity and noted that this may have introduced discrepancy between their participants' self-reports and the video assessment criteria, thus reducing their calculated accuracy. We reported a weak relationship $(\kappa 0.24)$ between sleep-onset and waking position via PSG-determination, whereas previous studies have reported discrepant results-McIntyre et al reported no relationship $(\kappa-0.13)$ via video determination, and the Auckland Stillbirth Study reported a strong relationship (Pearson's r 0.72) via self-reports ${ }^{2}$; however, our study has shown self-reports of sleeponset and waking position to have only moderate and weak agreement with the gold standard (PSG), respectively, which points to reliance on non-PSG methods (eg, self-reports) as a potential source of this discrepancy.

Warland and Dorrian reported a three-night observational study of 30 healthy women in late pregnancy and found moderate basic correlation between self-reported and video-determined time on the left $(\mathrm{r}=0.418)^{8}$; however, they did not report correlations for right or supine sleep. We report a good correlation between self-reported and
PSG-determined time on the left (Spearman's r 0.76 , $\mathrm{p}<0.01)$. Notably, we found poor correlation between self-reports and PSG-determined supine time and that pregnant women tend to underestimate this value. There are only two other studies investigating reliability of adults' self-reports of sleep position ${ }^{45}{ }^{46}$; both consist of non-pregnant adults, have conflicting results with each other, and are not reported in adequate detail for comparison to our results.

In the non-pregnant population, approximately $20 \%$ of a typical night's sleep is spent in the REM state and $80 \%$ is spent in the NREM state. The sleep architecture in the current study is consistent with previously reported patterns during pregnancy, that is, reduced slow-wave sleep, REM sleep and sleep efficiency. ${ }^{47-51}$

\section{Meaning of the study}

The previous studies by Owusu et al and O'Brien and Warland $^{6}$ recommended the development and testing of a PT device for pregnant women. In our study, a PT intervention was implemented in a population of healthy, third-trimester, pregnant women during sleep in a clinical sleep laboratory environment over two nights. This study extends the work of Stacey et $a l^{2}{ }^{2}$ Gordon et $a l^{3}$ McCowan et $a l^{4}$ and Warland and Mitchell ${ }^{52}$ by finding that supine sleep position in late pregnancy can be reduced with PT.

Left-side sleeping is common among pregnant women. This may be a more comfortable position but also may be related to the 'sleep-on-side' messages prevalent on the internet and among maternity care providers. Women in our study preferentially settled to sleep on their left for $73 \%$ of the nights and right for $20 \%$ of the nights. This is corroborated by McCowan et at who reported a significant increase in left-sided going-to-sleep position ( $43 \%$ to $58 \%)$ and small decrease in supine going-to-sleep position (5\% to $3.8 \%)$ in New Zealand over an approximate 5-year period following publicity of The Auckland Stillbirth Study by Stacey et $a l^{2}{ }^{2}$ If the supine sleeper is to be identified by history taking and stratified for targeted education or intervention, obstetricians, midwives and nurses should be aware that despite sleep-on-side knowledge and high rates of lateral going-to-sleep position, most pregnant women continue to spend a significant amount of time supine during sleep in late pregnancy per our study and previous studies. ${ }^{6-8}$ Also, per our study, pregnant women's estimates of time in each sleep position, while relatively accurate for lateral sleeping positions, underestimate the time they spend supine. Together, this suggests that healthcare providers may need to recommend interventions to prevent unintentional supine positioning during sleep.

\section{Strengths}

Strengths of this study include its rigorous methodology and use of the gold-standard sleep diagnostic test in a controlled laboratory setting. The sham intervention ensured that on the sham PSG night, participants received any specific benefit of any element of the PrenaBelt above 
and beyond all benefits that might be attributed to its ability to cause pressure points and, thus, reduced treatment bias. The crossover allowed each participant to serve as her own control for comparison of intervention effect on outcomes. Allocation concealment and randomisation of participants to intervention order helped avoid allocation bias and the potential impact of changes to sleep across the two PSG nights that could have resulted from familiarisation with the equipment and environment. Blinding of the participants, RAs, Registered PSG Technicians and the sleep specialist physician further reduced potential sources of bias and strengthened data integrity.

\section{Weaknesses}

Weaknesses of this study included that we had no baseline sleep data (run-in measurement) to which we could compare the PrenaBelt and sham nights; therefore, conclusions cannot be made regarding the effect, if any, of the PrenaBelt on sleep architecture and respiration in relation to no intervention. Participants' unfamiliarity with the sleep environment also may have affected sleep quantity and quality; however, we were unaware of any impact reported by participants. Period effects and carryover may have occurred due to advancing gestation and familiarisation with the sleep environment/equipment; however, given the median washout period (1 day) was negligible in the context of a 12-week third trimester, period effects from the washout were, at most, minimal. On the second PSG night, participants were exposed to the alternate intervention and could have unblinded themselves if they remembered what the intervention felt like on the first PSG night; however, we are unaware of what effect this may have had. The current study was conducted in a cohort of healthy, non-obese and majority Caucasian pregnant women. Due to the small sample size, this study may be underpowered and potentially overstate the true reduction in percentage of supine sleep; however, the sleep time in our study was relatively short (median 5.87 hours). Our participants reported a median of 8 hours overnight sleep duration at home; therefore, even if the reduction in supine sleep is lower than we observed, over a longer sleep duration, the PrenaBelt is still likely to result in a significant amount of supine sleep avoided. The power analysis was performed using the one-sided t-test, which could be a source of systemic confounding. Because of the fragility of our primary outcome ( $p$ 0.03), a confounding could shift our conclusions into statistical non-significance. This stresses the importance of future research to ascertain if our observed effects persist.

\section{Future research}

To date, it is unclear whether formal advice, given by a maternity care provider to a pregnant woman, about sleeping position in late pregnancy is less, as, or more effective in reducing supine sleep in comparison to a PT device such as the PrenaBelt. Future research comparing advice versus a PT intervention in the home setting is imperative. If sleeping supine is potentially harmful to the fetus, the amount of supine time that is harmful needs quantification in order to target interventions to avoid this. Given that estimations of time in each body position during sleep are based on the recollection of an unconscious state and thus inherently inaccurate, future research should not rely on self-reports but should incorporate an objective measure of body position during sleep throughout the third trimester allowing body position to be directly linked to pregnancy outcomes. The results of our randomised pilot trial warrant future, large, multiethnic studies that include women with a range of pregnancy and health conditions to ascertain if the observed effects persist.

\section{OTHER INFORMATION \\ Protocol}

Full details of the trial protocol are available with the full text of this article (see online supplementary file 1).

\section{Author affiliations}

${ }^{1}$ Dalhousie Medical School, Halifax, Nova Scotia, Canada

${ }^{2}$ Department of Obstetrics \& Gynaecology, University of Toronto, Toronto, Ontario,

Canada

${ }^{3}$ Global Innovations for Reproductive Health \& Life, Cleveland, Ohio, USA

${ }^{4}$ Department of Obstetrics and Gynecology, Dalhousie University, Halifax, Nova

Scotia, Canada

${ }^{5}$ Sleep Disorders Center, Department of Neurology, University of Michigan, Ann Arbor, Michigan, USA

${ }^{6}$ Department of Obstetrics and Gynecology, University of Michigan, Ann Arbor, Michigan, USA

${ }^{7}$ Georgetown University School of Medicine, Washington, District of Columbia, USA

${ }^{8}$ Dalhousie University, Halifax, Nova Scotia, Canada

${ }^{9}$ Innovative Canadians for Change, Edmonton, Alberta, Canada

${ }^{10}$ Method Squared Designhaus, Surrey, British Columbia, Canada

${ }^{11}$ Obstetrics and Gynaecology Department, Korle Bu Teaching Hospital, Accra, Ghana

${ }^{12}$ School of Medicine and Dentistry, University of Ghana, Accra, Ghana

${ }^{13}$ Department of Medicine, Dalhousie University, Halifax, Nova Scotia, Canada

${ }^{14}$ Nova Scotia Health Authority, Halifax, Nova Scotia, Canada

\section{Twitter@allankember}

Acknowledgements The authors would like to thank the 20 pregnant volunteers who gave up their time and the comfort of home to spend two nights in our study. Darlene Baxendale, Antonia Hall, Nooshin Sobhani and Laura Miller for their support as research assistants, Janet Slaunwhite for her administrative support and RPSGTs Antonya Schulz, Carrie Edwards-Young, Tracey Kent and Kelmarie Cole for their technical expertise in polysomnography setup and scoring. There were no patient advisers.

Contributors AJK designed the protocol, secured funding, ethics approval and the research contract, trained the study personnel, maintained the study master record, facilitated PSG studies as an unblinded personnel, monitored data collection, cleaned and analysed the data, and drafted and revised the paper. $\mathrm{He}$ is the guarantor. AJK and $\mathrm{AB}$ conceptualised the PrenaBelt. KC designed and manufactured the PrenaBelt research samples. DLM, HMS, LMO, AB, JC, MBB, $\mathrm{KC}$ and $\mathrm{Al}$ designed the protocol. HMS oversaw participant recruitment and was the principal investigator responsible for all aspects of the trial. DLM was the supervising investigator, oversaw the PSG studies at the trial site and interpreted the PSG studies. MBB wrote the statistical plan and oversaw the statistical analysis. JHW maintained the study master record, facilitated PSG studies as an unblinded personnel, monitored data collection, and cleaned and analysed the data. Al negotiated the research contract and managed the trial funds. DLM, HMS, LMO, AB, JC, JHW, MBB, KC and Al made intellectual contributions to the paper.

Funding This trial was supported by funding from Grand Challenges Canada Stars in Global Health Award, Round 7, Number 0629-01-10 and the IWK Health 
Centre Dr. H.B. Atlee Endowment Award. Grand Challenges Canada is funded by the Government of Canada.

Competing interests $A J K$ and $A B$ are officers at GIRHL, which has a patent application for the PrenaBelt (\#W02016176632A1) on which AJK, AB and KC are listed as inventors. DLM reports grants from Bresotec and grants from CIHR/ Industry Grant (Phillips) outside the submitted work. HMS, JC, JHW, MBB, Al and LMO have declared no support from any organisation for the submitted work, no financial relationships with any organisations that might have an interest in the submitted work in the previous three years, and no other relationships or activities that could appear to have influenced the submitted work.

Patient consent Not required.

Ethics approval This trial was approved by the IWK Health Centre Research Ethics Board in June 2015 (Project No. 1018753).

Provenance and peer review Not commissioned; externally peer reviewed.

Data sharing statement Extra data can be accessed via the Dryad data repository at http://www.datadryad.org/with the doi:10.5061/dryad.qg1b2n0.

Open access This is an open access article distributed in accordance with the Creative Commons Attribution Non Commercial (CC BY-NC 4.0) license, which permits others to distribute, remix, adapt, build upon this work non-commercially, and license their derivative works on different terms, provided the original work is properly cited, appropriate credit is given, any changes made indicated, and the use is non-commercial. See: http://creativecommons.org/licenses/by-nc/4.0/.

\section{REFERENCES}

1. Owusu JT, Anderson FJ, Coleman J, et al. Association of maternal sleep practices with pre-eclampsia, low birth weight, and stillbirth among Ghanaian women. Int J Gynaecol Obstet 2013;121:261-5.

2. Stacey T, Thompson JM, Mitchell EA, et al. Association between maternal sleep practices and risk of late stillbirth: a case-control study. BMJ 2011;342:d3403.

3. Gordon A, Raynes-Greenow C, Bond D, et al. Sleep position, fetal growth restriction, and late-pregnancy stillbirth: the Sydney stillbirth study. Obstet Gynecol 2015;125:347-55.

4. McCowan LME, Thompson JMD, Cronin RS, et al. Going to sleep in the supine position is a modifiable risk factor for late pregnancy stillbirth; Findings from the New Zealand multicentre stillbirth casecontrol study. PLoS One2017;12:e0179396.

5. Heazell A, Li M, Budd J, et al. Association between maternal sleep practices and late stillbirth - findings from a stillbirth case-control study. BJOG 2018;125:254-62.

6. O'Brien LM, Warland J. Typical sleep positions in pregnant women. Early Hum Dev 2014;90:315-7.

7. Mclntyre JP, Ingham CM, Hutchinson BL, et al. A description of sleep behaviour in healthy late pregnancy, and the accuracy of self-reports. BMC Pregnancy Childbirth 2016;16:115.

8. Warland J, Dorrian J. Accuracy of self-reported sleep position in late pregnancy. PLoS One 2014;9:e115760.

9. Courtney L. Supine hypotension syndrome during caesarean section. Br Med J 1970;1:797-8.

10. Holmes F. Spinal analgesia and caesarean section; maternal mortality. J Obstet Gynaecol Br Emp 1957;64:229-32.

11. Holmes F. The supine hypotensive syndrome. Anaesthesia 1995;50:972-7.

12. Holmes $F$. Incidence of the supine hypotensive syndrome in late pregnancy. A clinical study in 500 subjects. J Obstet Gynaecol Br Emp 1960;67:254-8.

13. Kauppila A, Koskinen M, Puolakka J, et al. Decreased intervillous and unchanged myometrial blood flow in supine recumbency. Obstet Gynecol 1980;55:203-5.

14. Kinsella SM, Lee A, Spencer JA. Maternal and fetal effects of the supine and pelvic tilt positions in late pregnancy. Eur J Obstet Gynecol Reprod Biol 1990;36(1-2):11-17.

15. Kinsella SM, Lohmann G. Supine hypotensive syndrome. Obstet Gynecol 1994;83(5 Pt 1):774-88.

16. Kuo CD, Chen GY, Yang MJ, et al. The effect of position on autonomic nervous activity in late pregnancy. Anaesthesia1997;52:1161-5.

17. Lee SW, Khaw KS, Ngan Kee WD, et al. Haemodynamic effects from aortocaval compression at different angles of lateral tilt in nonlabouring term pregnant women. Br J Anaesth 2012;109:950-6.

18. Lees MM, Scott DB, Kerr MG, et al. The circulatory effects of recumbent postural change in late pregnancy. Clin Sci 1967;32:453-65.
19. Lees MM, Taylor SH, Scott DB, et al. A study of cardiac output at rest throughout pregnancy. J Obstet Gynaecol Br Commonw 1967;74:319-28.

20. Milsom I, Forssman L. Factors influencing aortocaval compression in late pregnancy. Am J Obstet Gynecol 1984;148:764-71.

21. Rossi A, Cornette J, Johnson MR, et al. Quantitative cardiovascular magnetic resonance in pregnant women: crosssectional analysis of physiological parameters throughout pregnancy and the impact of the supine position. J Cardiovasc Magn Reson 2011;13:31.

22. Sharwood-Smith G, Drummond GB. Hypotension in obstetric spinal anaesthesia: a lesson from pre-eclampsia. Br J Anaesth 2009;102:291-4

23. Khatib N, Haberman S, Belooseski R, et al. 701: Maternal supine recumbency leads to brain auto-regulation in the fetus and elicit the brain sparing effect in low risk pregnancies. Am J Obstet Gynecol 2011;204:S278.

24. Carbonne $B$, Benachi $A$, Lévèque $M L$, et al. Maternal position during labor: effects on fetal oxygen saturation measured by pulse oximetry. Obstet Gynecol 1996;88:797-800.

25. Humphries A, Stone P, Mirjalili SA. The collateral venous system in late pregnancy: A systematic review of the literature. Clin Anat 2017;30:1087-95.

26. Cartwright RD. Effect of sleep position on sleep apnea severity. Sleep 1984;7:110-4.

27. Oksenberg A. Positional and non-positional obstructive sleep apnea patients. Sleep Med 2005;6:377-8.

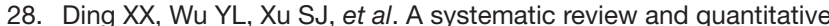
assessment of sleep-disordered breathing during pregnancy and perinatal outcomes. Sleep Breath 2014;18:703-13.

29. O'Brien LM, Bullough AS, Owusu JT, et al. Snoring during pregnancy and delivery outcomes: a cohort study. Sleep 2013;36:1625-32.

30. O'Brien LM, Bullough AS, Owusu JT, et al. Pregnancy-onset habitual snoring, gestational hypertension, and preeclampsia: prospective cohort study. Am J Obstet Gynecol 2012;207:487.e1-9.

31. Oksenberg A, Gadoth N. Are we missing a simple treatment for most adult sleep apnea patients? The avoidance of the supine sleep position. J Sleep Res 2014;23:204-10.

32. Ravesloot MJ, van Maanen JP, Dun L, et al. The undervalued potential of positional therapy in position-dependent snoring and obstructive sleep apnea-a review of the literature. Sleep Breath 2013;17:39-49.

33. Eldridge SM, Lancaster GA, Campbell MJ, et al. Defining Feasibility and Pilot Studies in Preparation for Randomised Controlled Trials: Development of a Conceptual Framework. PLoS One 2016;11:e0150205

34. Berger M, Oksenberg A, Silverberg DS, et al. Avoiding the supine position during sleep lowers $24 \mathrm{~h}$ blood pressure in obstructive sleep apnea (OSA) patients. J Hum Hypertens 1997;11:657-64.

35. Permut I, Diaz-Abad M, Chatila W, et al. Comparison of positional therapy to CPAP in patients with positional obstructive sleep apnea. $J$ Clin Sleep Med 2010;6:238-43.

36. Eijsvogel MM, Ubbink R, Dekker J, et al. Sleep position trainer versus tennis ball technique in positional obstructive sleep apnea syndrome. J Clin Sleep Med 2015;11:139-47.

37. Berry RB, Brooks R, Gamaldo CE, et al. The AASM Manual for the Scoring of Sleep and Associated Events: Rules, Terminology and Technical Specifications, Version 2.2. Internet]. Darien, Illinois 2015.

38. Cohen J. A Power Primer. Psychol Bull 1992;112.

39. Core Team R. R: A language and environment for statistical computing. Vienna, Austria: R Foundation for Statistical Computing, 2016.

40. van Maanen JP, Meester KA, Dun LN, et al. The sleep position trainer: a new treatment for positional obstructive sleep apnoea. Sleep Breath 2013;17:771-9.

41. Heinzer RC, Pellaton C, Rey V, et al. Positional therapy for obstructive sleep apnea: an objective measurement of patients' usage and efficacy at home. Sleep Med 2012;13:425-8.

42. van Maanen JP, de Vries N. Long-term effectiveness and compliance of positional therapy with the sleep position trainer in the treatment of positional obstructive sleep apnea syndrome. Sleep 2014;37:1209-15.

43. Bignold JJ, Mercer JD, Antic NA, et al. Accurate position monitoring and improved supine-dependent obstructive sleep apnea with a new position recording and supine avoidance device. J Clin Sleep Med 2011;7:376-83.

44. Zaremba S, Mueller N, Heisig AM, et al. Elevated upper body position improves pregnancy-related OSA without impairing sleep quality or sleep architecture early after delivery. Chest 2015;148:936-44.

45. Russo K, Bianchi MT. How Reliable Is Self-Reported Body Position during Sleep? J Clin Sleep Med 2016;12:127-8. 
46. Gordon SJ, Grimmer KA, Trott P, et al. Self-Reported versus Recorded Sleep Position: An Observational Study. J Allied Heal Sci Pract 2004;2.

47. Pien GW, Schwab RJ. Sleep disorders during pregnancy. Sleep 2004;27:1405-17.

48. Wilson DL, Barnes M, Ellett L, et al. Decreased sleep efficiency, increased wake after sleep onset and increased cortical arousals in late pregnancy. Aust N Z J Obstet Gynaecol 2011;51:38-46.
49. Schorr SJ, Chawla A, Devidas M, et al. Sleep patterns in pregnancy: a longitudinal study of polysomnography recordings during pregnancy. J Perinatol 1998;18(6 Pt 1):427-30.

50. Hertz $\mathrm{G}$, Fast $\mathrm{A}$, Feinsilver $\mathrm{SH}$, et al. Sleep in normal late pregnancy. Sleep 1992;15:246-51.

51. Petre-Quadens O, De Barsy AM, Devos J, et al. Sleep in pregnancy: evidence of foetal-sleep characteristics. J Neurol Sci 1967;4:600-5.

52. Warland J, Mitchell EA. A triple risk model for unexplained late stillbirth. BMC Pregnancy Childbirth 2014:14:142. 